Deutsches Exilarchiv 1933-1945 und Sammlung Exil-Literatur 1933-1945 
Die Deutsche Bibliothek, Leipzig, Frankfurt am Main, Berlin

\section{Deutsches Exilarchiv 1933-1945 und Sammlung Exil-Literatur 1933-1945}

Katalog der Bücher und Broschüren

Zugleich Band 2 von

Deutsches Exilarchiv 1933-1945:

Katalog der Bücher und Broschüren (1989) 
Redaktionsschluss: 14.3 .2002

Bearbeiterin: Mechthild Hahner

Wissenschaftliche Leitung: Brita Eckert

Bibliographische Information Der Deutschen Bibliothek Die Deutsche Bibliothek verzeichnet diese Publikation in der Deutschen Nationalbibliographie; detaillierte bibliographische Daten sind im Internet über <http://dnb.ddb.de $>$ abrufbar

ISBN 978-3-476-01939-4

ISBN 978-3-476-02924-9 (eBook)

DOI 10.1007/978-3-476-02924-9

Dieses Werk einschließlich aller seiner Teile ist urheberrechtlich geschützt. Jede Verwertung außerhalb der engen Grenzen des Urheberrechtsgesetzes ist ohne Zustimmung des Verlages unzulässig und strafbar. Das gilt insbesondere für Vervielfältigungen, Übersetzungen, Mikroverfilmungen und die Einspeicherung und Verarbeitung in elektronischen Systemen.

(C) 2003 Springer-Verlag GmbH Deutschland

Ursprünglich erschienen bei J. B. Metzlersche Verlagsbuchhandlung

und Carl Ernst Poeschel Verlag GmbH in Stuttgart 2003

www.metzlerverlag.de

info@metzlerverlag.de 


\section{Inhaltsverzeichnis}

Vorwort ..................... . . VII

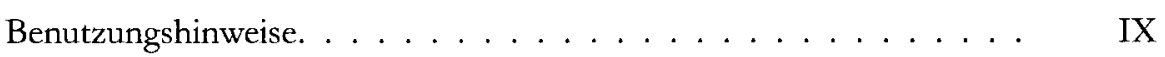

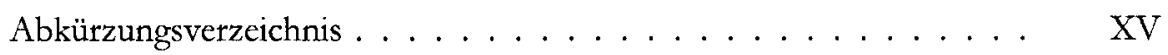

Hauptteil . . . . . . . . . . . . . . . . . . 1

Register . . . . . . . . . . . . . . . . . . . 571

Sonstige beteiligte Personen und Organisationen . . . . . . . . 571

In Widmungen und Besitzvermerken zusätzlich genannte

Emigranten und Exilorganisationen . . . . . . . . . . . 587

Verlage und verlegende Stellen . . . . . . . . . . . . . . . 588

Sachregister ....................... 610 


\section{Vorwort}

Beim Erscheinen des Katalogs der Bücher und Broschüren des Deutschen Exilarchivs 1933-1945 im Jahre 1989, ${ }^{1}$ der den Bestand bis einschließlich des Zugangsjahres 1985 verzeichnet, wurden für die Anzeige der späteren Erwerbungen Supplementbände angekündigt, die etwa im Fünfjahresabstand herauskommen sollten. Stattdessen legen wir nun nach längerer Zeit einen weiteren Band vor, der an Umfang den ersten nahezu erreicht. Dieses Vorgehen wurde durch die Folgen der erfreulichen politischen Entwicklung unseres Landes nahe gelegt.

Mit der Einigung Deutschlands am 3. Oktober 1990 wurden die Deutsche Bücherei Leipzig und die Deutsche Bibliothek Frankfurt am Main zu der Institution Die Deutsche Bibliothek zusammengefasst; die neue Einrichtung verfügt über zwei Exilsammlungen - neben dem Deutschen Exilarchiv 1933-1945 der Deutschen Bibliothek in Frankfurt am Main auch über die Sammlung Exil-Literatur 1933-1945 der Deutschen Bücherei in Leipzig.

Beide Exilsammlungen haben bei der Rezeption der Exilliteratur in den beiden deutschen Staaten jeweils eine erhebliche Rolle gespielt. Da über ihre Entstehung und Entwicklung mehrfach - und oft ausführlich - berichtet worden ist, kann hier auf die entsprechende $\mathrm{Li}$ teratur verwiesen werden. ${ }^{2}$

Nach der Vereinigung der beiden Häuser durch den Einigungsvertrag wurde beschlossen, das Modell der Arbeitsteilung, das für die Neuerscheinungen gilt, auch auf das Gebiet der Exilveröffentlichungen zu übertragen; dies bedeutet die Fortführung der Sammlungen in beiden Häusern, die bibliografische Verzeichnung einer Publikation jedoch nur in einem Haus.

Beide Exilsammlungen werden, vor allem durch antiquarische Käufe, ständig erweitert, soweit es die Haushaltsmittel erlauben. Die bibliografische Verzeichnung geschieht arbeitsteilig: Während das Deutsche Exilarchiv für die Verzeichnung der Monografien zuständig ist, widmet sich die Sammlung Exil-Literatur der Erschließung der Periodika; ein gemeinsames Bestandsverzeichnis der Exilzeitschriften und -zeitungen unter Leipziger Redaktion ist in Vorbereitung.

Ein erstes Ergebnis der Arbeitsteilung und Zusammenarbeit der Exilsammlungen auf bibliografischem Gebiet ist der vorliegende Bestandskatalog, der in Frankfurt aufgrund von Autopsie - auch der Leipziger Bestände - erarbeitet wurde. Wegen der erheblichen Anzahl der anzuzeigenden Monografien erschien es uns sinnvoll, anstelle von Supplementen einen umfangreichen Band vorzulegen, der zwei Funktionen erfüllt:

Er ist die Fortsetzung des 1989 erschienenen "Katalogs der Bücher und Broschüren« des Deutschen Exilarchivs 1933-1945 der Deutschen Bibliothek (im Folgenden "Band 1 « genannt) mit den Neuerwerbungen der Jahre 1986 bis 1995 und damit Band 2 dieses Bestandskatalogs. Gleichzeitig ist er der Bestandskatalog der Sammlung Exil-Literatur 1933-1945 der Deutschen Bücherei aller bis Ende 1995 erworbenen Monografien, soweit die Titel nicht in Band 1 verzeichnet sind. Er enthält somit Bücher und Broschüren, die entweder nur in Leipzig oder nur in Frankfurt oder aber in beiden Exilsammlungen vorhanden sind. Dies wird durch den der Signatur vorgestellten Besitzvermerk: »DBL« und/oder "DBF « deutlich.

Zusammen verzeichnen die beiden Bände des »Katalogs der Bücher und Broschüren« alle Monografien der beiden Exilsammlungen bis zum Erwerbungsjahr 1995. Da aus Gründen der Übersichtlichkeit das Prinzip galt, jedes Buch nur einmal zu verzeichnen, kann jedoch nicht nachgewiesen werden, welche der in Band 1 verzeichneten Titel in der Leipziger Sammlung vorhanden sind. Es ist aber geplant, die Titel des inzwischen nahezu vergriffenen 1. Bandes als geschlossenen Bestand innerhalb des Online-Benutzerkatalogs Der Deutschen Bibliothek zu präsentieren, der dann auch die Leipziger Besitzvermerke tragen wird.

Die Aufnahmekriterien sind gegenüber Band 1 unverändert geblieben: Verzeichnet werden von Emigranten verfasste Werke in Erstausgaben, Nachauflagen und Übersetzungen sowie Sammelbände, an denen Emigranten mitgearbeitet haben; darüber hinaus von Emigranten herausgegebene, übersetzte, illustrierte und gestaltete Bücher. Hinzu kommt die gesamte Produktion von Exilverlagen. Auch die Veröffentlichungen jüdischer Verlage und Organisationen in Deutschland, Österreich und der Tschechoslowakei nach 1933 wurden verzeichnet, da sie in einem winneren Exil entstanden sind und oft der Vorbereitung der Emigration dienten. Aufgenommen wurden auch für die illegale Arbeit in Deutschland hergestellte Tarnschriften, die meist im Exil verfasst und gedruckt wurden. (Ausführlicher s.a. "Benutzungshinweise«, S. IX.)

Wie das Sachregister zeigt, enthält Band 2 - wie Band 1 - nach wie vor besonders zahlreiche Veröffentlichungen aus den Gebieten der Belletristik und der politischen Publizistik, obwohl wir beim Erscheinen des 1. Bandes davon ausgegangen waren, dass hierbei Vollständigkeit nahezu erreicht sei, zumindest was die einzelnen Werke in der Originalsprache betrifft. In den folgenden zehn Jahren wurden jedoch in Frankfurt wie in Leipzig zahlreiche Übersetzungen und weitere Auflagen und Ausgaben bereits verzeichneter Werke erworben; hinzu 
kommt, dass durch die Aufnahme der Leipziger "Altbestände« und durch gezielte Suche im Internet nach Büchern, die lange auf unseren Desideratenlisten standen, zusätzliche Werke aus Belletristik und politischer Publizistik verzeichnet werden konnten. - Angezeigt werden aber auch zahlreiche Veröffentlichungen aus den Bereichen der jüdischen Emigration und vor allem der Wissenschaftsemigration, die seit Jahren einen Erwerbungsschwerpunkt beider Sammlungen bildet. Neben den Kulturwissenschaften - vor allem Geschichte, Kunstwissenschaften, Literaturwissenschaft, Musikwissenschaft, Sozialwissenschaften, Politologie, Theologie und Philosophie - sind nun auch die Naturwissenschaften, Mathematik, Medizin, Psychologie, die Rechtswissenschaft und die Wirtschaftswissenschaften mit zahlreichen Monografien vertreten.

Der Hauptteil des 2. Bandes enthält mit rund 5550 Katalogeintragungen, die im Anschluss an Band 1 weiternummeriert wurden (Nummern 6908-12419), etwa $80 \%$ des Umfangs des 1. Bandes (rund 6900 Eintragungen). Zusätzlich wurden einige Titel aus Band 1 wiederholt, weil sich vor allem Änderungen in der physischen Form ergaben (Originale statt der dort angezeigten Mikrofilme). Unveränderte Nachauflagen erhalten keinen eigenen Eintrag, sondern werden nur als Fußnote unter dem Ersteintrag angegeben.

In Band 1 und 2 zusammen dürfte der weltweit umfangreichste Bestand von Büchern und Broschüren des deutschsprachigen Exils der Jahre 1933 bis 1945 bibliografisch verzeichnet worden sein.

Das Deutsche Exilarchiv 1933-1945 besaß Ende des Jahres 200217270 Bücher und Broschüren, außerdem 9621 Zeitschriftenbände (bzw. -hefte) von rund 1000

\section{Anmerkungen}

1. Deutsches Exilarchiv 1933-1945. Katalog der Bücher und Btoschüren. Redaktionsschluss: 31.12.1985. Redaktion: Mechthild Hahner. Wissenschaftliche Leitung: Werner Berthold u. Brita Eckert. - Stuttgart: Metzler, 1989. XIX, 714 S. (Sonderveröffentlichungen der Deutschen Bibliothek; Nr. 16).

2. Zur Geschichte der Exilsammlung der Deutschen Bibliothek in Frankfurt a.M. siehe u. a.:

Werner Berthold: Exilliteratur und Exilforschung. Ausgewählte Aufsätze, Vorträge und Rezensionen. Mit einer Einleitung von Wolfgang Frühwald. Hrsg. von Brita Eckert u. Harro Kieser. Wiesbaden: Harrassowitz, 1996. 211 S. (Gesellschaft für das Buch; Bd. 3);

35 Jahre Exilliteratur 1933-1945 in der Deutschen Bibliothek Frankfurt am Main. Ein Beitrag zur Geschichte der Exilforschung in der Bundesrepublik Deutschland. Für Werner Berthold zum 31. März 1984. Eine Ausstellung der Deutschen Bibliothek, Frankfurt am Main. Ausstellung u. Katalog: Brita Eckert, Mechthild Hahnet u. a. - Frankfurt a. M.: Deutsche Bibliothek, 1984. VIII, 233 S. (Sonderveröffentlichungen der Deutschen Bibliothek; Nr. 13);
Periodika; die Sammlung Exil-Literatur 1933-1945 10016 Bände und 16835 Zeitschriftenstücke (Hefte).

Die Differenz zwischen den vorhandenen und den in den beiden Katalogen verzeichneten Büchern (zur Zeit des Redaktionsschlusses Ende 1995 wurden im Deutschen Exilarchiv 14825 Bücher gezählt, in der Sammlung Exil-Literatur 8555 Monografien) ergibt sich aus der Tatsache, dass beide Sammlungen auch Zweitexemplare enthalten, die aufgrund des schlechten Erhaltungszustandes eines vorhandenen Exemplars nachgekauft wurden, darüber hinaus ergänzendes, für die Forschung unentbehrliches Material, wie zeitgenössische Veröffentlichungen des Auslandes zum deutschsprachigen Exil, Publikationen deutschsprachiger Emigranten, die nach 1950 erschienen sind, oder nationalsozialistische Schriften. In den Bestandskatalog wurden jedoch nur die nach den exakten Kriterien als Exilveröffentlichungen definierten Bücher und Broschüren aufgenommen (s. Benutzungshinweise, S. IX).

Der »Katalog der Bücher und Broschüren« des Deutschen Exilarchivs 1933-1945 wurde bald nach seinem Erscheinen zum unentbehrlichen Handbuch für Exilforscher, Antiquare und andere am Thema Exil, der Literatur des 20. Jahrhunderts und anderen Themen Interessierte. Seit langem wird von vielen Seiten die Fortsetzung angemahnt. Wir legen sie hiermit vor und hoffen, dass dieser Band die gleiche positive Aufnahme finden möge wie der erste und vielfach nützlich sein wird.

Frankfurt am Main, im Mai 2003

Elisabeth Niggemann Generaldirektorin

Die Deutsche Bibliothek
Brita Eckert: Das Deutsche Exilarchiv 1933-1945. In: Nationalbibliotheken im Jahr 2000. (Festgabe für Günther Pflug zum 65. Geburtstag.) Hrsg. von Sieghardt von Köckritz u. Kurt Nowak unter Mitarb. von Maria Daab. - Frankfurt a. M.: BuchhändlerVereinigung, 1988, S. 265-[291].

Zur Geschichte der Exilsammlung der Deutschen Bücherei Leipzig siehe u. a.:

Horst Halfmann: Das Schrifttum der Emigration in der Deutschen Bücherei. In: Deutsche Bücherei 1912-1962. Festschrift zum fünfzigjährigen Bestehen der Deutschen Nationalbibliothek. Redaktion: Hclmut Rötzsch u.a. - Leipzig: Deutsche Bücherei 1962, S. 197-217;

Jörg Räuber: Exil-Literatur. Die Deutsche Bibliothek Frankfurt a. M. \& Leipzig. In: Kulturen im Kontext. Zehn Jahre Sammlung Deutscher Drucke. Hrsg. von der Staatsbibliothek zu Berlin Preußischer Kulturbesitz im Auftrag der Arbeitsgemeinschaft Sammlung Deutscher Drucke. - Wiesbaden: Reichert, 1999, S. $142-163$. 


\section{Benutzungshinweise}

Inhalt

Der Bestandskatalog der Bücher und Broschüren des Deutschen Exilarchivs 1933-1945 und der Sammlung Exil-Literatur 1933-1945 verzeichnet Publikationen deutschsprachiger Emigranten, die in den Jahren 1933-1945 im Ausland erschienen sind; aufgenommen wurden auch im Ausland erschienene Veröffentlichungen von 1945 bis 1950, wenn der entsprechende Autor in diesem Zeitraum nicht nach Deutschland zurückgekehrt ist. Im Einzelnen handelt es sich um

a. von Emigranten verfasste Bücher in allen Ausgaben und Auflagen

b. Übersetzungen von Büchern deutschsprachiger Emigranten

c. Sammelbände, an denen Emigranten mitgearbeitet haben

d. von Emigranten herausgegebene und/oder eingeleitete Veröffentlichungen

e. von Emigranten illustrierte und/oder gestaltete Bücher

f. von Emigranten übersetzte Bücher

g. die Produktion von Exilverlagen oder -organisationen

Der Katalog enthält außerdem:

h. Veröffentlichungen jüdischer Verleger und Or-

Anlage

Der Bestandskatalog der Bücher und Broschüren des Deutschen Exilarchivs 1933-1945 und der Sammlung Exil-Literatur 1933-1945 besteht aus einem alphabetischen Titelverzeichnis (Hauptteil) und vier Registern.

\section{Hauptteil}

\section{Aufnahme der Titel}

Die Titel werden nach den »Regeln für die alphabetische Katalogisierung « (RAK) unter dem persönlichen Verfasser, dem korporativen Verfasser oder, bei anonymen Schriften und Werken mit mehr als drei Verfassern, unter dem Sachtitel verzeichnet.

Die »Regeln für die alphabetische Katalogisierung« wurden vor allem für Bibliothekskataloge konzipiert. Im Hinblick auf die bibliografische Verzeichnung und unter Berücksichtigung von Besonderheiten des hier zu verzeichnenden Materials wurden gegenüber den »Regeln« ganisationen in Deutschland, Österreich und in der Tschechoslowakei nach 1933

i. Veröffentlichungen nicht bzw. noch nicht emigrierter in Deutschland lebender Autoren zwischen 1933 und 1945 im Ausland

k. Veröffentlichungen nicht oder noch nicht emigrierter deutschsprachiger Autoren in Österreich, in der Tschechoslowakei u. a. Ländern von 1933 an, von denen mit Gewissheit oder hoher Wahrscheinlichkeit anzunehmen ist, dass ihnen reichsdeutsche Publikationsmöglichkeiten versperrt waren (z. B.: Irene Harand: „Sein Kampf«. - Wien 1935).

Als Kriterium für die Gruppen $\mathrm{i}$ und $\mathrm{k}$ gilt das Verbot oder die Behinderung freier Publikationsmöglichkeiten im deutschen Machtbereich.

In den Bestandskatalog aufgenommen wurden die $\mathrm{Zu}$ gänge des Deutschen Exilarchivs in Frankfurt von 1986 bis 1995 und der Bestand der Sammlung Exil-Literatur in Leipzig bis einschließlich Erwerbungsjahr 1995, soweit diese Titel nicht in dem 1989 erschienenen Band der Bücher und Broschüren des Deutschen Exilarchivs verzeichnet sind. verschiedene Veränderungen und Vereinfachungen eingeführt:

So treten Verweisungen, d. h. Kurzhinweise auf die entsprechende Haupteintragung für Personen und Körperschaften, die ein Werk erarbeitet oder veranlasst und herausgegeben bzw. als Übersetzer, Illustratoren o. ä. an ihm beteiligt sind, an die Stelle von Nebeneintragungen mit der Wiederholung des gesamten Textes einer Eintragung. Nebeneintragungen innerhalb des Eintrags für einen Autor entfallen ersatzlos. Dies gilt für alle Emigranten bzw. Exilorganisationen. Andere beteiligte Personen und Organisationen werden im Register "Sonstige beteiligte Personen und Organisationen« erfasst.

Bei Verweisungen, die sich auf die Beteiligung eines Emigranten an einer Publikation beziehen, wird die Art der Beteiligung am Ende der Verweisung in eckigen Klammern angegeben, z. B. [1ll.], [Übers.], [Bearb.].

Die Form der Titelaufnahme entspricht den Richtlinien der »International Standard Bibliographic Description 
(ISBD)«. Die Aufnahmen beginnen mit der halbfett gesetzten Ansetzungsform des ersten persönlichen Verfassers bei Verfasserwerken oder der ersten Körperschaft bei Urheberwerken bzw. bei Sachtitelwerken mit der des Sachtitels. Dann folgt die eigentliche Titelbeschreibung, die die in der Vorlage enthaltenen Angaben in der international vereinbarten standardisierten Reihenfolge und Form wiedergibt.

Vergleiche Beispiele am Ende der Hinweise.

Schreibung und Orthografie der Vorlage werden in der bibliografischen Beschreibung im Allgemeinen beibehalten.

Für die Groß- und Kleinschreibung gelten, unabhängig von der Typografie der Vorlage, die Rechtschreiberegeln der betreffenden Sprache.

Nach den »Regeln für die alphabetische Katalogisierungu erhalten Schriftenreihen (Serien) eine eigene Titelaufnahme. Darunter werden die Stücktitel in der Abfolge der Serienzählung, bei ungezählten Serien nach dem Alphabet der Verfassernamen, verkürzt aufgeführt. Diese Regel wurde in dem vorliegenden Katalog nur angewandt auf Schriftenreihen, in denen überwiegend Emigranten publiziert haben, auf Serien, die von Emigranten herausgegeben oder verlegt wurden, sowie auf Schriftenreihen jüdischer Verlage und Organisationen in Deutschland, Österreich und der Tschechoslowakei nach 1933 (s. o.). Bei Schriftenreihen, in denen nur einzelne Exilwerke erschienen sind, wird der Serientitel nur bei den Einzelstücken aufgeführt. Nach dem gleichen Prinzip wird bei mehrbändigen Werken eines Verfassers mit Aufnahmen für die einzelnen Bände (»Stücktitelaufnahmen«) bei gesammelten Werken u. ä. verfahren, d.h. eine Gesamttitelaufnahme des mehrbändigen Werkes erfolgt nur bei Exilautoren.

Am Ende der Titelaufnahme werden Erläuterungen zum Inhalt, der Erscheinungsform usw. als Fußnoten in kleinerem Schriftgrad aufgeführt. Dabei werden bei der Angabe des Inhalts von Sammelbänden nur die Beiträge von Emigranten berücksichtigt.

Unveränderte Neuauflagen erhalten keine eigene Aufnahme; sie werden nach der ersten vorhandenen Ausgabe in einer Fußnote aufgefühtt.

\section{Ansetzung der Titel}

Die Ansetzung der Titel erfolgt im Hinblick auf ihre Einordnung. Für die Ordnung der Eintragungen werden die dafür heranzuziehenden Teile (Namen von Personen und Körperschaften, Sachtitel) angesetzt, d.h. einordnungsfähig gemacht.

In Eigennamen und Körperschaftsnamen werden alle
Wörter außer Artikeln, Präpositionen und Konjunktionen im Innern der Namen groß geschrieben. Anführungszeichen entfallen hierbei.

Das erste Wort eines Sachtitels, auch eines zitierten Sachtitels, wird groß geschrieben.

Nichtlateinische Schriften sind gemäß den in wissenschaftlichen Bibliotheken gültigen Instruktionen transliteriert.

\subsection{Ansetzung persönlicher Verfasser}

Verfasser und sonstige beteiligte Personen werden unter der von ihnen selbst gebrauchten Namensform angesetzt. Bei mehrgliedrigen Familiennamen entscheidet die Gepflogenheit des Staates, dessen Bürger die Person ist, über die Ansetzungsform.

Artikel und Präpositionen können daher - je nach Landesgebrauch - als Bestandteile des Familiennamens am Anfang des Namens bei der Ansetzung berücksichtigt oder nicht berücksichtigt werden. Berücksichtigte Artikel und Präpositionen werden zusammen mit dem folgenden Namensbestandteil als ein Ordnungswort behandelt. Nicht berücksichtigte Artikel und Präpositionen stehen als nicht ordnende Bestandteile am Ende der Namen.

Franz von Rintelen $=$ Rintelen, Franz von [Deutscher]

Hubertus Prinz zu Löwenstein = Löwenstein, $\mathrm{Hu}$ bertus Prinz zu [Deutscher]

Mark Van Doren $=$ VanDoren, Mark [Amerikaner] C. P. de la Fournière $=$ LaFournière, C. P. de [Franzose]

Vincent van Gogh $=$ Gogh, Vincent van [Niederländer]

Verwandtschaftsbezeichnungen vor Familiennamen, wie Fitz, Mac und O', werden zum Namen gezogen und, wenn sie abgekürzt sind, zur vollen Form ergänzt.

Thomas Watson MacCallum = MacCallum, Thomas Watson

Mary O'Hara = O'Hara, Mary

Doppelnamen können entweder unverbunden oder durch Bindestriche, Präpositionen, Artikel oder Konjunktionen verbunden sein. Artikel und Präpositionen im Innern von Familiendoppelnamen werden in Bezug auf den zweiten Namensbestandteil analog zu den am Anfang eines mehrgliedrigen Namens stehenden Artikeln und Präpositionen behandelt: Sind sie Bestandteil des zweiten Namensteiles, bilden sie zusammen mit diesem ein Ordnungswort, andernfalls ist jeder Teil eines Doppelnamens, der durch ein Spatium von einem anderen Namensteil getrennt ist, ein eigenes Ordnungswort.

Morstein Marx $=2$ Ordnungswörter

Faber DuFaur $=2$ Ordnungswörter 
Vieth von Golssenau $=3$ Ordnungswörter aber

Berg-Andrée $=1$ Ordnungswort

Coudenhove-Kalergi $=1$ Ordnungswort

Vornamen unterschiedlicher Schreibung werden nicht vereinheitlicht, es sind also unterschieden:

Müller, Carl, und Müller, Karl

Antike und mittelalterliche Autoren sind unter den Vornamen gestellt.

Musaeus < Poeta>

Leonardo $<$ da Vinci $>$

Raffaello $<$ Sanzio $>$

\subsection{Ansetrung von Körperschaften}

Körperschaften werden im Allgemeinen unter ihrem offiziellen Namen angesetzt.

Freie Deutsche Bühne <Buenos Aires>

Reichsvertretung der Deutschen Juden

Körperschaften, die anderen unterstellt oder zugehörig sind und die nicht ohne den Namen der übergeordneten Körperschaft zitiert werden können, werden als Abteilung der übergeordneten Körperschaft, und zwar durch einen Schrägstrich von dieser getrennt, angesetzt.

Reichsvertretung der Juden in Deutschland / Abteilung Wirtschaftshilfe

\subsection{Ansetzung von Sachtiteln}

Der Sachtitel wird im Allgemeinen in der vorliegenden Form angesetzt. Sind einzelne Bestandteile nicht einordnungsgerecht, wird die Einordnungsform der Vorlageform in eckigen Klammern nachgestellt. Ein Ansetzungstitel - in eckigen Klammern stehend - wird gebildet, wenn die vorliegenden Formen (Wörter, Ziffern, Zeichen), die nicht einordnungsfähig sind, überwiegen oder die Anzahl der einordnungsfähigen Bestandteile eines Sachtitels gleich ist.

Das 3. Reich in der Karikatur = Das [Dritte] Reich in der Karikatur

3 Jahre F.D.B. $=$ [Drei Jahre Freie Deutsche Bühne]

9. November $1938=$ [Neunter November neunzehnhundertachtunddreißig]

\section{Ordnung der Titel}

Die Titel sind in einem Alphabet nach persönlichen Verfassern und Körperschaften geordnet; bei Sachtitelwerken ist der Sachtitel für die Einordnung maßgebend.

Frei, Bruno [persönl. Verf.]

Frei-Deutschland-Bewegung [Körperschaft]
Freie Deutsche Jugend [Körperschaft]

Freiheit und Volk [Sachtitel]

Freimann, Aron [persönl. Verf.]

Maßgebend für die Ordnung der Eintragungen ist die Ordnung nach der gegebenen Wortfolge. Lediglich ein am Anfang eines Sachtitels oder eines Körperschaftsnamens stehender bestimmter oder unbestimmter Artikel, gleich welchen Falles, und ein ihm gleichlautendes Zahlwort oder Pronomen sowie am Schluss von Verfassernamen stehende Präpositionen, Artikel und Adelstitel werden übergangen. Bei Verfasser- und Urheberwerken wird daher auf die besondere Kennzeichnung des ersten Ordnungswortes des Sachtitels verzichtet. Zu beachten ist, dass bei der Ordnung nach der gegebenen Wortfolge Buchstabenfolgen nur durch ein nach Rechtschreiberegeln zu setzendes Spatium in mehrere Ordnungswörter getrennt werden. Wortzusammensetzungen mit Bindestrich gelten demgemäß als ein Ordnungswort.

Kedem-Taschen-Wörterbuch $=1$ Ordnungswort

Welt-Jugend-Woche $=1$ Ordnungswort

Eine Folge von Initialen gilt als ein Ordnungswort, z. B. FGLC, ISK, KC, VSBD. - Die Buchstaben ä, ö sowie ø und ü werden wie ae, oe und ue geordnet.

\subsection{Ordnung der Werke persönlicher Verfasser}

Die Werke eines persönlichen Verfassers werden alphabetisch nach den vorliegenden Sachtiteln geordnet. In Abweichung von den »Regeln für die alphabetische $\mathrm{Ka}$ talogisierung« werden Übersetzungen eines Exilautors nicht unter dem Titel der Übersetzung, sondern unter dem Einordnungssachtitel (Originaltitel) nach dem Alphabet der Sprachen verzeichnet. Die Sprachbezeichnung steht kursiv neben der Katalog-Nummer. Sofern für einen Einheitssachtitel keine Ausgabe vorhanden ist, wird vor der ersten Übersetzung der Titel der Erstausgabe mit dem Erscheinungsjahr in eckigen Klammern angegeben. Verweisungen vom vorliegenden Titel entfallen.

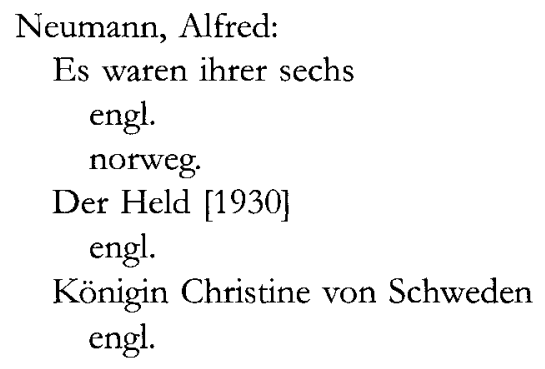

\subsection{Ordnung von Urbeberwerken}

Die Veröffentlichungen einer Körperschaft werden nach dem vorliegenden Sachtitel geordnet. 
Bei gleichnamigen Körperschaften steht diejenige ohne Ordnungshilfe vor einer solchen mit einer Ordnungshilfe; gleichnamige Körperschaften ohne Abteilung stehen vor solchen mit einer Abteilung.

Frei-Deutschland-Bewegung: Goals Jüdische Gemeinde $<$ Berlin>: Verwaltungsbericht Jüdische Gemeinde <Berlin> / Aufbringungswerk Reichsvertretung der Juden in Deutschland: Arbeitsbericht

Reichsvertretung der Juden in Deutschland / Abteilung Wirtschaftshilfe

Die bibliografischen Beschreibungen (Titelaufnahmen) der einzelnen Veröffentlichungen sind nummeriert; dabei wurde die Zählung von Band 1 weitergeführt. Die Katalog-Nummer steht links über der Titelaufnahme. Katalog-Nummern in runden Klammern, die die fortlaufende Nummerierung unterbrechen, beziehen sich auf Titel des ersten Bandes, die hier wiederholt werden, weil sich Änderungen ergeben haben:

(701) Bredel, Willi: Der Spitzel... (Der Mikrofilm wurde durch ein Original ersetzt);

(6194) Was soll mit den Juden geschehen? (als Bearbeiter konnte Hans Siemsen ermittelt werden)

Nicht gezählt werden die Aufnahmen der Schriftenreihen und Gesamttitelaufnahmen von mehrbändigen Werken.

\section{Inhaltliche und typografische Besonderheiten}

Bei der Namensansetzung von Exilautoren wird in Form einer Kurzbezeichnung auf die entsprechenden
Artikel im "Biographischen Handbuch der deutschsprachigen Emigration nach 1933 «* verwiesen, soweit sie dort in Band 1 oder 2 vertreten sind (Abkürzung: $\mathrm{BHb}$ 1, BHb 2).

Dann folgen Angaben über abweichende Namensformen, Namensänderungen und Pseudonyme. In der Regel werden nur die Namen aufgeführt, zu denen Veröffentlichungen vorliegen.

Bei Autoren, unter denen z.T. oder überwiegend Titel der Gruppen h-k (Veröffentlichungen jüdischer Verlage und Organisationen in Deutschland, Österreich und in der Tschechoslowakei nach 1933 usw.) vorliegen, ist nach Möglichkeit das Jahr der Emigration angegeben.

Bei allen Publikationen wurde auf der gleichen Zeile mit der Katalog-Nummer rechtsbündig die Signatur der Deutschen Bibliothek (DBF) und/oder der Deutschen Bücherei (DBL) angegeben.

Publikationen der Gruppen $\mathrm{h}-\mathrm{k}$ (Veröffentlichungen jüdischer Verlage und Organisationen in Deutschland, Österreich und in der Tschechoslowakei nach 1933 usw.) werden durch einen Stern rechts neben der Katalog-Nummer gekennzeichnet.

Bei Werken, die nicht wegen ihres Autors, sondern aufgrund emigrierter, sonstiger (am Buch) beteiligter Personen oder Körperschaften (wie Mitarbeiter, Bearbeiter, Herausgeber, Übersetzer, Illustratoren, Buchgestalter) oder als Veröffentlichung eines emigrierten Verlages oder Verlegers aufgenommen werden, sind die entsprechenden Angaben (Bearbeiter, Übersetzer, Verlag u. a.) kursiv gesetzt. Treffen mehrere Kriterien zu, so ist in der Regel nur die erste Angabe durch Kursivsatz ausgezeichnet.

\footnotetext{
* Biographisches Handbuch der deutschsprachigen Emigration nach 1933 = International biographical dictionary of Central European emigrés 1933-1945. Hrsg. vom Institut für Zeitgeschichte, München, u. von der Research Foundation for Jewish Immigration, New York, unter der Gesamtleitung von Werner Röder u. Herbert A. Strauss. Bd. 1-3. - München u. a. 1980-1983.
} 
Beispiele:

\section{Mann, Thomas}

\begin{tabular}{|c|c|c|}
\hline \multirow{2}{*}{\multicolumn{3}{|c|}{ Katalog-Nummer $\quad 9976$ tschech. $/$ Sprachbezeichnung $^{\text {DBF: EB } 90 / 86 / /^{\text {Standort }} \text { Signatur }}$}} \\
\hline & & Katalog-Nummer $\quad 9976$ tschech. Sprachbezeichnung ${ }^{\text {DBF: EB } 90 / 86 / \text { Standort }}$ \\
\hline \multirow{2}{*}{\multicolumn{3}{|c|}{$\begin{array}{l}\text { Priběhy Jákobovy / Thomas Mann. [Prel. Ivan Olbracht } \\
\text { a Helena Malírova.] - Praha [Prag] : Melantrich, } 1934 .\end{array}$}} \\
\hline & & \\
\hline & - XLVII, 306 S. ; $19 \mathrm{~cm}$ & Seitenzahl, Format \\
\hline $\begin{array}{l}\text { Gesamttitelangabe }- \\
\text { Gesamttitelangabe }\end{array}$ & Josef a bratri jeho / Thor & \\
\hline \multirow{2}{*}{$\begin{array}{l}\text { Gesamttitelangabe } \\
\text { Einheitssachtitel }\end{array}$} & (Spisů /Thomas Mann ; Sv. 8) & \\
\hline & EST: Die Geschichten Jaakobs <tschech.> & Einbandart \\
\hline
\end{tabular}

\section{Mann, Thomas}

Katalog-Nummer

Sachtitel

Sonstige Personen

Auflage, Ausgabe

Seitenzahl

Gesamttitelangabe

Gesamttitelangabe

9988

Der junge Joseph : Roman / Thomas Mann.

$\mathrm{BHb} 2$

[Schutzumschlag nach Entwurf von Karl Walser.] -

16. -18. Aufl. - Wien : Bermann-Fischer, 1936. 337 S. ; $20 \mathrm{~cm}$

(Joseph und seine Brüder / Thomas Mann 2)

(Gesammelte Werke / Thomas Mann) Lw.

\section{Avdeenko, Aleksandr Ostapovič}

Katalog-Nummer
Sachtitel
Sonstige Personen

7059

Zusatz zum Sachtitel

Ich liebe : Roman / Alexander Awdejenko

DBF: EB 95/78 _ Signatur

[Autoris. Übers. aus d. Russ. von Olga Halpern.

Schutzumschlag von Jobn Hearffield.] -

Erscheinungsvermerk

Basel : Universum-Buchgemeinschaft, 1938. - 292 S. $20 \mathrm{~cm}$

Format

(Der internationale Roman: N. F. ; 1938, Bd. 1)

(Universum-Bücherei ; Bd. 281)

Schriftenreihe

EST: Ja liubliu <dt.> Lw.

\section{Register}

Die Register verweisen auf die Katalog-Nummern des Hauptteils.

1. Sonstige beteiligte Personen und Organisationen

Aufgeführt werden Herausgeber oder herausgebende Stellen, Illustratoren u.a., die keine Emigranten sind oder als solche bisher nicht zu ermitteln waren. Alle Emigranten und Exilorganisationen, die an einer Publikation als Herausgeber, Übersetzer, Illustratoren u.a. beteiligt sind, werden im Hauptteil verwiesen (s.a. 1. Aufnahme der Titel, S. IX). Dieses Register wurde erstellt, um den Hauptteil nicht zu umfangreich zu gestalten.

2. In Widmungen und Besitzvermerken zusätzlich genannte Emigranten und Exilorganisationen

Sie werden im Hauptteil nicht verwiesen.

3. Verlage und verlegende Stellen

Sie werden in ihrer vollständigen Namensform mit allen Verlagsorten angesetzt. Emigranten als Verlagsinhaber sind nach dem Verlagsnamen in eckigen Klammern angegeben. 


\section{Sachregister}

Das Sachregister bietet eine Hilfe für den inhaltlichen Zugriff. Aus personellen und zeitlichen Gründen war es jedoch nicht möglich, die Exilsammlungen nach den jetzt von Der Deutschen Bibliothek angewendeten »Regeln für den Schlagwortkatalog« zu bearbeiten.

Die einzelnen Formal- und Sachgruppen des Registers wurden nach rein pragmatischen Gesichtspunkten festgelegt. Sie ergeben sich zum Teil aus dem vorliegenden Material (z. B. »Politische Publizistik: Tarnschriften«, »Von Emigranten gestaltete Bücher«), teilweise richten sie sich auch nach Fragestellungen der Exilforschung (z. B. "Belletristik: Historische Romane und Erzählungen«). Den Schwerpunkt der Sammlung und damit auch des Registers bilden auch im vorliegenden Band belletristische und politische Texte, wie die Gruppe »Literatur, Politik (Texte)« zeigt.
Bei der Gruppe "Einzelne Sachgebiete und Wissenschaften« wurde die wissenschaftliche mit der populärwissenschaftlichen Literatur zusammen erfasst; deshalb wird sowohl die Bezeichnung für das Sachgebiet wie die Bezeichnung für die Wissenschaft angegeben (z.B. »Musik, Musikwissenschaft ()).

Enthält eine Publikation mehrere Aspekte, für die im Sachregister Einordnungsstellen vorgesehen sind, wird sie mehrfach verzeichnet.

Publikationen, an denen Emigranten nur als Übersetzer oder Illustratoren bzw. Gestalter beteiligt waren, werden nur in den Formalgruppen »Von Emigranten übersetzte Bücher« und »Von Emigranten gestaltete bzw. illustrierte Bücher« erfasst. Veröffentlichungen, die nur in den Katalog aufgenommen werden, weil sie in einem Exilverlag erschienen sind, erhalten nur im Verlagsregister einen Eintrag.

Hinweis:

Die Bücher und Broschüren des Deutschen Exilarchivs in Frankfurt und der Sammlung Exil-Literatur in Leipzig werden, entsprechend dem Charakter Der Deutschen Bibliothek als Archivbibliothek, aus Gründen des Bestandsschutzes nicht in den Fernleihverkehr gegeben. Sie können nur in den Leseräumen eingesehen werden. 


\section{Abkürzungsverzeichnis}

\begin{tabular}{|c|c|c|c|}
\hline a.A. & am Albis & D. C., DC & District of Columbia \\
\hline a.d.E. & an der Eger & d.h. & das heißt \\
\hline a.M. & am Main & D.F. & Distrito Federal \\
\hline a.N. & am Neckar & d.i. & das ist \\
\hline a.Rh. & am Rhein & dän. & dänisch \\
\hline $\mathrm{AB}$ & Aktiebolaget & Darst. & Darstellung \\
\hline Abt. & Abteilung & dass. & dasselbe \\
\hline add. & addition(s) & $\mathrm{DBF}$ & Deutsche Bibliothek Frankfurt a.M. \\
\hline afterw. & afterword & DBL & Deutsche Bücherei Leipzig \\
\hline AG & Aktiengesellschaft & DEA & Deutsches Exilarchiv 1933-1945 \\
\hline AIA & Artists' International Association & $\operatorname{dep}(t)$ & department \\
\hline akad. & akademisch & Dez. & Dezember \\
\hline amerikan. & amerikanisch & Diagr. & Diagramm(e) \\
\hline Anh. & Anhang & Diss. & Dissertation \\
\hline Anm. & Anmerkung(en) & Dra & Doktora \\
\hline AS & Aktieselskabet & DSAP & Deutsche Sozialdemokratische Arbeiterpartei \\
\hline Aufl. & Aufl. & dt. & deutsch \\
\hline Aufn. & Aufnahme(n) & durchgearb. & durchgearbeitet \\
\hline Auftr. & Auftrag & durchges. & durchgesehen \\
\hline Ausg. & Ausgabe & & \\
\hline ausgew. & ausgewählt(e) & e. & ein, eine, einer \\
\hline Ausw. & Auswahl & ECCI & Executive Committee of the Communist \\
\hline Ausz. & Auszug, Auszüge & & International \\
\hline autoris. & autorisiert(e) & ed. & edited, edition, editor \\
\hline & & Einf. & Einführung \\
\hline BBC & British Broadcasting Corporation & eingel. & eingeleitet \\
\hline $\mathrm{Bd}$ & Band & Einl. & Einleitung \\
\hline Bdch. & Bändchen & EK & Exekutivkomitee \\
\hline bearb. & bearbeitet & EKKI & Exekutivkomitee der Kommunistischen \\
\hline Bearb. & Bearbeiter & & Internationale \\
\hline begr. & begründet & engl. & englisch \\
\hline beigef. & beigefügt & ENI & Éditions Nouvelles Internationales \\
\hline Beil. & Beilage(n) & erg. & ergänzt \\
\hline Beitr. & Beitrag, Beiträge & Erg. & Ergänzung \\
\hline berecht. & berechtigt(e) & Erg.-Bd. & Ergänzungsband \\
\hline bes. Berücks. & besondere Berücksichtigung & erl. & erläutert \\
\hline $\mathrm{BHb}$ & Biographisches Handbuch & Erl. & Erläuterung(en) \\
\hline bibliograph. & bibliographisch & Erstausg. & Erstausgabe \\
\hline biograph. & biographisch & erw. & erweitert(e) \\
\hline B1. & Blatt, Blätter & ESI & Éditions Sociales Internationales \\
\hline brit. & britisch & EST & Einheitssachtitel \\
\hline bros. & brothers & estn. & estnisch \\
\hline brosch. & broschiert & evang. & evangelisch \\
\hline bulgar. & bulgarisch & & \\
\hline bzw. & beziehungsweise & Fac. & Faculté \\
\hline & & FAI & Federación Anarquista Iberica \\
\hline c & Copyright & Fak. & Fakultät \\
\hline Č. & čast & Faks. & Faksimile(s) \\
\hline Calif. & California & Faltbl. & Faltblatt, Faltblätter \\
\hline CALPO & Comité Allemagne Libre Pour l'Ouest & Falttab. & Falttabelle(n) \\
\hline $\mathrm{CC}$ & Central Committee & Falttaf. & Falttafel(n) \\
\hline CCG & Control Commission Germany & FAM & Free Austrian Movement \\
\hline $\mathrm{cm}$ & Centimeter & farb. & farbig \\
\hline CN'T & Confederación Nacional del 'Trabajo & Fasc. & Fasciculus \\
\hline Co. & Company & FDKB & Freier Deutscher Kulturbund \\
\hline collab. & collaboration & FDR & Franklin Delano Roosevelt \\
\hline Conn. & Connecticut & ff. & folgende \\
\hline Čsl. & Cechoslovakisch & FGLC & Free German League of Culture in \\
\hline ČSR & Československá Republika & & Great Britain \\
\hline $\mathrm{CV}$ & Central-Verein (der Juden in Deutschland) & FNA & Front National Autrichien \\
\hline & & förf:s & författarens \\
\hline d. & der, die, das & ford. & fordidotta \\
\hline D. & Deel, Del & forew. & foreword \\
\hline
\end{tabular}




\begin{tabular}{|c|c|c|c|}
\hline $\begin{array}{l}\text { Forts. } \\
\text { franz. }\end{array}$ & $\begin{array}{l}\text { Fortsetzung } \\
\text { französisch }\end{array}$ & $\begin{array}{l}\text { Kt. } \\
\text { Kt. }\end{array}$ & $\begin{array}{l}\text { Kanton } \\
\text { Karte(n) }\end{array}$ \\
\hline \multirow[t]{2}{*}{ Galcit } & Guggenheim Aeronautical Laboratory, & lat. & lateinisch \\
\hline & California Institute of Technology & lett. & lettisch \\
\hline ganzs. & ganzseitig(e) & LID & League for Industrial Democracy \\
\hline geb. & geboren $(\mathrm{e})$ & literar. & literarisch \\
\hline \multirow[t]{2}{*}{ geh. } & gehalten & Literaturverz. & Literaturverzeichnis \\
\hline & geheftet & Ltd. & Limited \\
\hline Geleitw. & Geleitwort & Lw. & Leinwand \\
\hline Ges. & Gesellschaft & & \\
\hline gest. & gestorben & M.P. & Member of Parliament \\
\hline getr. Pag. & getrennte Paginierung & Mass. & Massachusetts \\
\hline Glos. & Gloucestershire & Mich. & Michigan \\
\hline $\mathrm{GmbH}$ & Gesellschaft mit beschränkter Haftung & Mij & Maatschappij \\
\hline graph. Darst. & graphische Darstellung(en) & Minn. & Minnesota \\
\hline griech. & griechisch & Mitarb. & Mitarbeit, Mitarbeiter \\
\hline & & Mitverf. & Mitverfasser \\
\hline $\mathrm{H}$. & Heft & ms. & maschinenschriftlich \\
\hline Hauptsacht. & Hauptsachtitel & Ms. & Manuskript \\
\hline Hauptt. & Haupttitel & mutmaßl. & mutmaßlich \\
\hline hebr. & hebräisch & & \\
\hline histor. & historisch & N.C. & North Carolina \\
\hline Hlw. & Halbleinwand & N.F. & Neue Folge \\
\hline holländ. & holländisch & N.J., NJ & New Jersey \\
\hline hrsg. & herausgegeben & N.Y., NY & New York \\
\hline Hrsg. & Herausgeber & Nachtr. & Nachtrag \\
\hline \multirow[t]{2}{*}{ hs. } & handschriftlich & Nachw. & Nachwort \\
\hline & & nat. & national \\
\hline i.e. & id est [das ist] & NCLC & National Council of Labour Colleges \\
\hline Ill. & Illinois & Nebent. & Nebentitel \\
\hline Ill. & Illustration(en), Illustrator & Neuausg. & Neuausgabe \\
\hline $\operatorname{imp}(r)$ & imprimerie & Neuaufl. & Neuauflage \\
\hline impr. & impression & niederländ. & niederländisch \\
\hline Inc. & Incorporated & No. & Number \\
\hline Ind. & Indiana & norweg. & norwegisch \\
\hline ING & Inside Nazi Germany & Notenbeisp. & Notenbeispiele \\
\hline Inst. & Institut & Nov. & November \\
\hline ISK & Internationaler Sozialistischer Kampfbund & Nr. & Nummer \\
\hline isländ. & isländisch & nrf & Nouvelle revue française \\
\hline \multirow[t]{2}{*}{ Isotype } & International system of typographic picture & & \\
\hline & education & o.ä. & oder ähnliche(s) \\
\hline ital. & italienisch & o.Nr. & ohne Nummer \\
\hline \multirow[t]{2}{*}{ Izdat. } & Izdatel'stvo & Obb. & Oberbayern \\
\hline & & Oct. & October \\
\hline Jg. & Jahrgang & österr. & österreichisch \\
\hline jidd. & jiddisch & övers. & översättning \\
\hline jr. & junior & Opl. & Oplag \\
\hline jüd. & jüdisch & $\begin{array}{l}\text { Orig. } \\
\text { orthod. }\end{array}$ & $\begin{array}{l}\text { Original } \\
\text { orthodox }\end{array}$ \\
\hline kart. & kartoniert & overs. & oversat, oversatt \\
\hline kath. & katholisch & Overs. & Oversaettelse \\
\hline Kat.-Nr. & Katalog-Nummer & & \\
\hline \multirow[t]{2}{*}{$\mathrm{KC}$} & Kartell-Convent der Verbindungen Deutscher & P. & Part \\
\hline & Studenten Jüdischen Glaubens & $\mathrm{Pa}$. & Pennsylvania \\
\hline KI & Kommunistische Internationale & PEN & (International Association of) Poets, Playwrights, \\
\hline KJI & Kommunistische Jugendinternationale & & Editors, Essayists, Novelists \\
\hline $\mathrm{KKL}$ & Keren Kajemeth Lejisrael & per. & perevod \\
\hline köt. & kötet & persönl. & persönlich \\
\hline Komm. & Kommission & philos. & philosophisch \\
\hline KPD & Kommunistische Partei Deutschlands & Pp. & Pappe \\
\hline $\mathrm{KPdSU}(\mathrm{B})$ & $\begin{array}{l}\text { Kommunistische Partei der Sowjetunion } \\
\text { (Bolschewiki) }\end{array}$ & Pr. & Press \\
\hline KPÖ & $\begin{array}{l}\text { (Bolschewik1) } \\
\text { Kommunistische Partei Österreichs }\end{array}$ & $\begin{array}{l}\text { predisl. } \\
\text { pref. }\end{array}$ & $\begin{array}{l}\text { predislovie } \\
\text { preface }\end{array}$ \\
\hline kroat. & kroatisch & préf. & préface \\
\hline
\end{tabular}




\begin{tabular}{|c|c|c|c|}
\hline přel. & prèložil & u.ä. & und ähnliche(s) \\
\hline print. & printed, printing & u.d.T. & unter dem Titel \\
\hline Pseud. & Pseudonym & udg. & udgave, udgives, udgivet \\
\hline publ. & published, publishing & $\mathrm{U}(\mathrm{d}) \mathrm{SSR}$ & Union der Sozialistischen Sowjet-Republiken \\
\hline Publ. Co. & Publishing Company & $\begin{array}{l}\text { überarb. } \\
\text { übers. }\end{array}$ & $\begin{array}{l}\text { überarbeitet } \\
\text { übersetzt }\end{array}$ \\
\hline R.I., RI & Rhode Island & Übers. & Übersetzer, Übersetzung(en) \\
\hline red. & redigiert & übertr. & übertragen \\
\hline Red. & Redakteur, Redaktion & Übertr. & Übertragung \\
\hline repr. & reprint, reprinted & uitg. & uitgave, uitgeverij \\
\hline rev. & revidiert, revised & Uitgeversmij. & Uitgeversmaatschappij \\
\hline \multirow[t]{3}{*}{ RSFSR } & Rossiskaja Sovetskaja Federativnaja & ukrain. & ukrainisch \\
\hline & Socialističeskaja Respublika $=$ & umgearb. & umgearbeitet \\
\hline & Russische Sozialistische Föderative Sowjetrepublik & ungar. & ungarisch \\
\hline Rücks. & Rückseite & Univ. & Universität, Université, University \\
\hline rumän. & rumänisch & Univ. Pr. & University Press \\
\hline \multirow[t]{2}{*}{ russ. } & russisch & unveränd. & unverändert(e) \\
\hline & & unvollst. & unvollständig \\
\hline s. & siehe & uppl. & upplaga \\
\hline S. & Seite(n) & USA & United States of America \\
\hline s.a. & siehe auch & USSR & Union of Socialist Soviet Republics \\
\hline \multirow{3}{*}{ S.A. } & Société Anonyme & usw. & und so weiter \\
\hline & Sociedad Anónima & & \\
\hline & Sociedade Anónima & Vegaar & Verlagsgenossenschaft Ausländischer Arbeiter \\
\hline s.l. & sine loco [ohne Ort] & & in der UdSSR \\
\hline s.n. & sine nomine [ohne Namen] & verantwortl. & verantwortlich \\
\hline s.o. & siehe oben & verb. & verbessert(e) \\
\hline Sacht. & Sachtitel & Verb. & Verbindung \\
\hline SAP & Sozialistische Arbeiterpartei Deutschlands & Verf. & Verfasser \\
\hline schwed. & schwedisch & Verl. & Verlag \\
\hline schweizer. & schweizerisch & Verl.-Anst. & Verlagsanstalt \\
\hline Selbstverl. & Selbstverlag & Verl.-Ges. & Verlagsgesellschaft \\
\hline Sept. & September & verm. & vermehrt(e) \\
\hline Ser. & Serie; series & vermutl. & vermutlich \\
\hline serb. & serbisch & vers. & versehen \\
\hline serbokroat. & serbokroatisch & vert. & vertaald, vertaling \\
\hline Sonderdr. & Sonderdruck & Verz. & Verzeichnis \\
\hline Sonderh. & Sonderheft & vgl. & vergleiche \\
\hline Sp. & Spalte(n) & vielm. & vielmehr \\
\hline SPD & Sozialdemokratische Partei Deutschlands & Vol. & Volume \\
\hline SSSR & Sojus Sovetskich Socialističeskich Respublik & vollst. & vollständig \\
\hline St. & Saint, Sankt & Vorw. & Vorwort \\
\hline Suppl. & Supplement & VSBD & Volkssozialistische Bewegung Deutschlands \\
\hline sv. & svazek & $\begin{array}{l}\text { Vt. } \\
\text { vyd. }\end{array}$ & $\begin{array}{l}\text { Vermont } \\
\text { vydání }\end{array}$ \\
\hline $\mathrm{T}$. & Teil & vyp. & Vypusk \\
\hline Tab. & Tabelle(n) & & \\
\hline Taf. & Tafel(n) & WAC & West-Alpen-Club \\
\hline tatar. & tatarisch & wahrscheinl. & wahrscheinlich \\
\hline techn. & technisch & WETUC & Workers' Educational Trade Union Committee \\
\hline Teilausg. & Teilausgabe & wirkl. Name & wirklicher Name \\
\hline teilw. & teilweise & Wis. & Wisconsin \\
\hline Tenn. & Tennessee & WIZO & Women's International Zionist Organization \\
\hline Tip. & Tipografia & & \\
\hline \multirow[t]{3}{*}{ trad. } & tradução & z.B. & zum Beispiel \\
\hline & traducción & z.T. & zum Teil \\
\hline & traduction, traduit & zahlr. & zahlreich $(\mathrm{e})$ \\
\hline transl. & translated, translation & Zch. & Zürich \\
\hline tschech, & tschechisch & Zeichn. & Zeichnung(en) \\
\hline tschechoslowak. & tschechoslowakisch & zionist. & zionistisch \\
\hline Tsd. & Tausend & $\mathrm{ZK}$ & Zentralkomitee \\
\hline \multirow[t]{2}{*}{ türk. } & türkisch & Zsarb. & Zusammenarbeit \\
\hline & & zsgest. & zusammengestellt \\
\hline $\mathrm{u}$. & und & zugl. & zugleich \\
\hline u.a. & und andere & ZVfD & Zionistische Vereinigung für Deutschland \\
\hline
\end{tabular}




\section{Kurzbezeichnungen für zitierte Werke}

BHb Biographisches Handbuch der deutschsprachigen Emigration nach 1933 = International biographical dictionary of Central European emigrés 19331945 / Leitung u. Bearb. : Werner Röder ; Herbert A. Strauss ... Bd. 1-3. - München [u.a.] : Saur, 1980-1983.

Siepmann Montage : John Heartfield : vom Club Dada zur Arbeiter-Illustrierten Zeitung; Dokumente, Analysen, Berichte / Eckhard Siepmann ... - 3., verb.
Aufl. - Berlin (West) : Elefanten Press Galerie, 1977.

St/T Deutsche Exil-Literatur 1933-1945 : eine Bio-Bibliographie / Wilhelm Sternfeld ; Eva Tiedemann. - 2., verb. u. stark erw. Aufl. - Heidelberg : L. Schneider, 1970.

(Veröffentlichungen der Deutschen Akademic für Sprache und Dichtung, Darmstadt ; Veröffentlichung 29 a) 